\title{
Estimation of subcellular organelle volume from ultrathin sections through centrioles with a discretized version of the nucleator.
}

\author{
Alexander A. Mironov, Galina V. Beznoussenko and Alexander A. Mironov Jr.*
}

Department of Cell Biology and Oncology, Consorzio "Mario Negri Sud", Via Nazionale, S. Maria Imbaro, 66030, Italy

Although immunocytochemistry, fluorescence microscopy and laser scanning confocal microscopy allow rather precise visualization of organelles such as mitochondria, nuclei, and large membrane compartments of the biosynthetic and endocytic pathways, their application for analysis of smaller structures (membrane compartments under $200 \mathrm{~nm}$ ) is rather limited due to low resolution. Therefore, in many cases the use of electron microscopy morphometry is unavoidable. Although the disector fits for the quantitation of rather big intracellular structures such as Golgi stacks, mitochondria, endosomes, vesiculo-tubular clusters (1) with sizes of at least 2-3-fold more than the thickness of ultrathin sections $(50-80 \mathrm{~nm})$, it is not suitable for evaluation of smaller structures due to the Holmes effect. Previously we proposed the centriole as a unique intracellular reference point suitable for local estimators such as the discretized rotator (2). Here we extend the applicability of this reference point for the nucleator. Our estimator represents a discretized version of the nucleator, where instead of measuring intercept lengths, the points in distance classes are counted and instead of vertical sections randomly oriented sections were explored. Centrioles, around which cells virtually rotate, serve as a unique reference point with a constant size allowing unbiased cell selection from the whole population with equal probability without the disector application.

RBL-2H3 cells were grown as described previously (2), fixed in warm $\left(37^{\circ} \mathrm{C}\right) 1 \%$ glutaraldehyde in 0.15M HEPES ( $\mathrm{pH} 7.2$ ) for 1-2 min and then left for $60 \mathrm{~min}$ at room temperature in the same fixative, cells were scrapped, centrifuged, washed in the same buffer and post-fixed in reduced $\mathrm{OsO}_{4}$ for $1 \mathrm{~h}$, dehydrated in ethanol, and embedded in Epon 812. After $12 \mathrm{~h}$ of polymerization at $60^{\circ} \mathrm{C}$ resin the pellets were divided into two parts at random angle and then control and experimental blocks were glued together with Epon 812 at $60^{\circ}$ C. Blocks were sectioned perpendicularly to the plane of glueing. Control and experimental cells with clearly identifiable centriole were photographed from the same "sandwich" section. For the discretized nucleator two test systems were used (Fig. 1). The first grid with circular lines with equal distances between them was

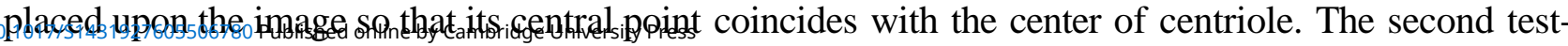


counted in each class (the area between two circles). Volume estimates of each compartment were calculated according to equation:

$$
\text { est } \mathrm{v}=\frac{\pi}{n} * a_{p} * \sum_{i=1}^{n} P_{i} * D_{i}
$$

where est $\mathrm{v}$ - estimate of total volume of structure, $\mathrm{a}_{\mathrm{p}}$ - area per one point, $\mathrm{P}_{\mathrm{i}}$ - number of points counted in one class, $\mathrm{D}_{\mathrm{i}}$ - the distance from each test point to the central point, $\mathrm{n}$ - number of centriolar sections measured. The precision of the estimator was explored by measuring volume of cells. The discretized nucleator gave practically identical results with the discretized rotator (Table 1) (3).

\section{References}

(1) S.I. Bannykh S.I., et al., J. Cell. Biol. 135 (1996) 19.

(2) A.A. Mironov Jr and A.A. Mironov A. A. J. Microsc. 192 (1998) 29.

(3) We thank Dr. A. Luini for helpful discussions and Dr. T. Tandrup for constructive advice. This work was supported by Telethon Italy (E1105; E0982), the Italian Association for Cancer Research (AIRC, Milano, Italy), the Italian National Research Council (CNR, Rome, Italy) Progetto Finalizzato "Biotecnologie" (ctr. n. 01.00035.PF49).

TABLE 1. Comparison of RBL cell volume estimates using different methods (mean \pm SD)

\begin{tabular}{ccccc}
\hline & Nucleator & Rotator & Discr. rotator & Discr. nucleator \\
\hline Cell volume, $\mu \mathrm{m}^{3}$ & $1440 \pm 500$ & $1508 \pm 383$ & $1527 \pm 364$ & $1540 \pm 380$ \\
\hline
\end{tabular}

FIG. 1. Scheme of the vertical discretized rotator procedure.

The volume of the cell (black countour) is equal to $\frac{\pi}{1} * a_{p} *(1 \times 1+6 \times 2+10 \times 3+8 \times 4+6 \times 5+4 \times 6+1 \times 7)$

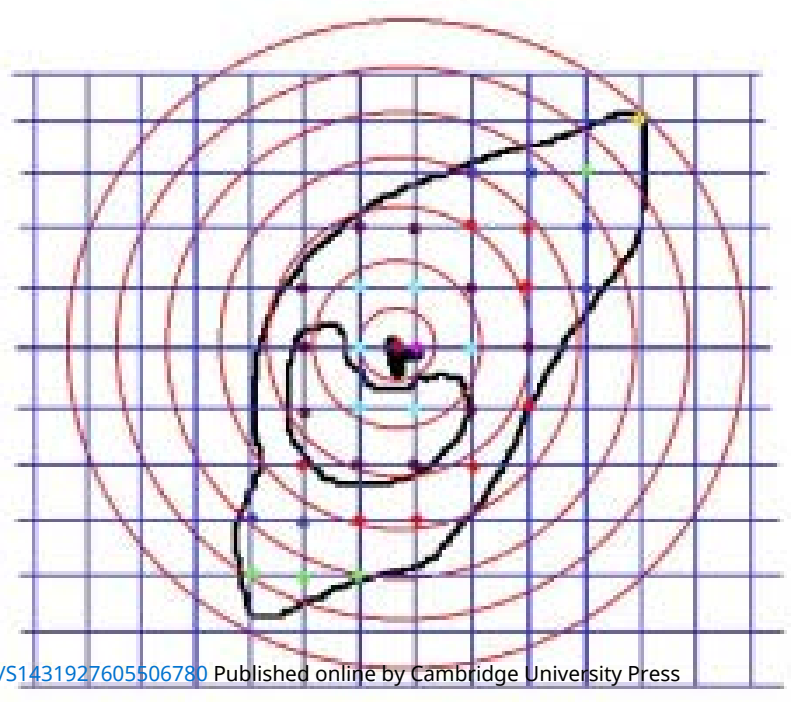

